\title{
Localized Dispersive States in Nonlinear Coupled Mode Equations for Light Propagation in Fiber Bragg Gratings*
}

\author{
C. Martel M. Higuera, and J. D. Carrasco
}

\begin{abstract}
Dispersion effects induce new instabilities and dynamics in the weakly nonlinear description of light propagation in fiber Bragg gratings. A new family of dispersive localized pulses that propagate with the group velocity is numerically found, and its stability is also analyzed. The unavoidable different asymptotic order of transport and dispersion effects plays a crucial role in the determination of these localized states. These results are also interesting from the point of view of general pattern formation since this asymptotic imbalance is a generic situation in any transport-dominated (i.e., nonzero group velocity) spatially extended system.
\end{abstract}

Key words. fiber Bragg gratings, nonlinear coupled mode equations, envelope equations, multiple scale methods, localized solutions

AMS subject classifications. $34 \mathrm{E} 13,74 \mathrm{~J} 30,74 \mathrm{~J} 34,37 \mathrm{~K} 45,78 \mathrm{~A} 60$

1. Introduction. Fiber Bragg gratings (FBG) are microstructured optical fibers that present a spatially periodic variation of the refractive index. The combination of the guiding properties of the periodic media with the Kerr nonlinearity of the fiber results in the very particular light propagation characteristic of these elements, which make them very promising for many technological applications that range from optical communications (wavelength division, dispersion management, optical buffers and storing devices, etc.) to fiber sensing (structural stress measure in aircraft components and buildings, temperature change detection, etc.); see, e.g., the recent review [12].

The amplitude equations that are commonly used in the literature to model one dimensional light propagation in an FBG are the so-called nonlinear coupled mode equations (NLCME) $[24,7,9,8,1,10]$, which, conveniently scaled, can be written as

$$
\begin{aligned}
& A_{t}^{+}-A_{x}^{+}=\mathrm{i} \kappa A^{-}+\mathrm{i} A^{+}\left(\sigma\left|A^{+}\right|^{2}+\left|A^{-}\right|^{2}\right), \\
& A_{t}^{-}+A_{x}^{-}=\mathrm{i} \kappa A^{+}+\mathrm{i} A^{-}\left(\sigma\left|A^{-}\right|^{2}+\left|A^{+}\right|^{2}\right),
\end{aligned}
$$

where $A^{ \pm}$are the envelopes of the two counterpropagating wavetrains that resonate with the grating, $\kappa$ is the strength of the coupling effect produced by the grating, and $\sigma>0$ is ratio of the self to cross nonlinear interaction coefficient $\left(\sigma=\frac{1}{2}\right.$ for a cubic Kerr nonlinearity [9]). 
The NLCME can be obtained from the full Maxwell-Lorentz equations using multiple scales techniques in the limit of small grating depth, small light intensity, and slow spatial and temporal dependence of the field envelopes (see [10] for a detailed description of this derivation process).

It has been recently shown $[16,17]$ that light propagation in FBG can develop dispersive structures that are not accounted for in the NLCME formulation, and that, to correctly describe the weakly nonlinear dynamics of the system, the NLCME have to be completed with material dispersion terms:

$$
\begin{aligned}
& A_{t}^{+}-A_{x}^{+}=\mathrm{i} \kappa A^{-}+\mathrm{i} A^{+}\left(\sigma\left|A^{+}\right|^{2}+\left|A^{-}\right|^{2}\right)+\mathrm{i} \varepsilon A_{x x}^{+}, \\
& A_{t}^{-}+A_{x}^{-}=\mathrm{i} \kappa A^{+}+\mathrm{i} A^{-}\left(\sigma\left|A^{-}\right|^{2}+\left|A^{+}\right|^{2}\right)+\mathrm{i} \varepsilon A_{x x}^{-} .
\end{aligned}
$$

The dispersive nonlinear coupled mode equations (NLCMEd) above are scaled as the NLCME: the characteristic length scale is the slow scale that results from the balance of the advection term with the small effect of the grating, the characteristic time is the corresponding transport time scale (which sets to one the scaled group velocity), and the characteristic size of the wavetrains is the one resulting from the saturation of the small nonlinear terms. The slow envelope assumption forces the dispersive terms to always be small as compared with the advection terms; in other words, second derivatives of the slow amplitudes are much smaller than their first derivatives. In the scaled equations this effect is contained in the scaled dispersion coefficient $\varepsilon$ (which measures the dispersion to transport ratio), and therefore, in order to be consistent with the slow envelope assumption, the NLCMEd must be considered in the limit $\varepsilon \rightarrow 0$. The NLCMEd were already analyzed in [6], but they considered only the case $\varepsilon \sim 1$ that, as explained above, does not correspond to a generic situation from the point of view of large scale pattern formation in extended systems.

The NLCMEd can be regarded as asymptotically nonuniform, in the sense that the NLCMEd is an asymptotic model obtained in the $\varepsilon \rightarrow 0$ (weakly nonlinear, slow envelope) limit that still contains the small parameter $\varepsilon$. This is the unavoidable consequence of simultaneously considering two balances of different asymptotic order: one induced by the dominant effect of the transport at the group velocity (balance described by NLCME) and a second one that is associated with the underlying dispersive, nonlinear, Schrödinger-like dynamics of the system. The small dispersive terms in the NLCMEd are essential to describe the dynamics of the system when it develops small dispersive scales $\delta_{\text {disp }} \sim \sqrt{|\varepsilon|}$. As it was shown in [16], the NLCMEd in the $\varepsilon \rightarrow 0$ limit constitute a singular perturbation problem (cf. [14]), and the onset of the dispersive scales is not a higher order, longer time effect; it takes place in the same time scale of the NLCME, no matter how small the dispersion coefficient $\varepsilon$ is.

A solution of the NLCMEd for $\varepsilon=-10^{-3}$ that exhibits small dispersive scales all over the domain is represented in Figure 1: note that, for short time, the dispersive structures just propagate with the group velocity, but, for $t \sim 1$, they also interact with each other, giving rise to a very complicated spatio-temporal pattern. The initial condition used in this simulation was a uniform modulus solution with a small and smooth (i.e., associated with an exponentially decaying spectrum) random perturbation that, according to the dispersionless NLCME formulation, was a stable solution (the details of the numerical integration method are given in [16]). In order to ensure that the small scales in Figure 1 are dispersion-induced 

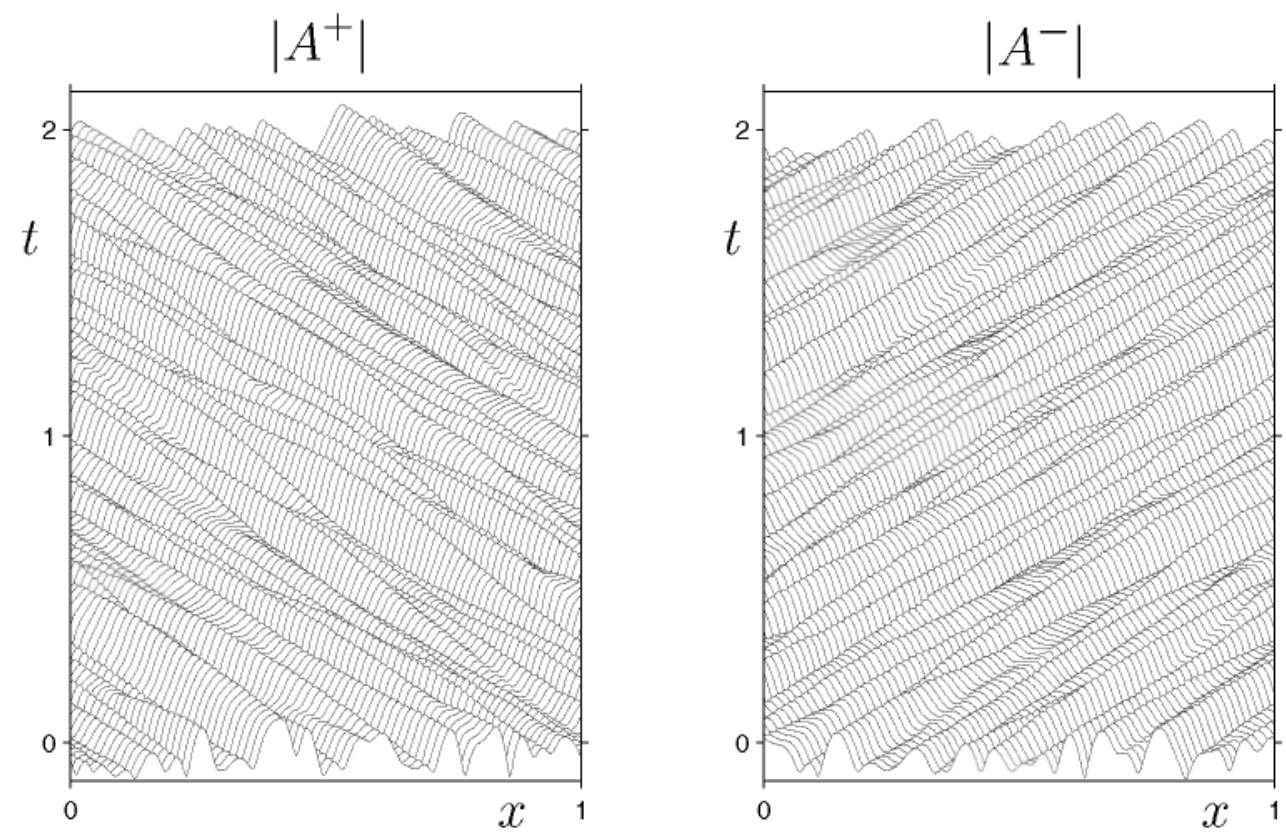

Figure 1. Space-time representation of a solution of the NLCMEd exhibiting small dispersive scales all over the domain $\left(\sigma=1 / 2, \kappa=2, \varepsilon=-10^{-3}\right.$, and periodicity boundary conditions). See the accompanying file (69822_01.gif [22.4MB]) for an animation of the onset of the dispersive scales.

scales we have repeated the NLCMEd simulation but with a reduced dispersion $\varepsilon=-10^{-3} / 4$. The result is plotted in Figure 2, where it can be seen that the small scales again fill the entire domain, but their typical size, $\delta_{\text {disp }} \sim \sqrt{|\varepsilon|}$, is now approximately one half of that in Figure 1 (see also the corresponding animations 69822_01.gif [22.4MB] and 69822_02.gif [20.1MB]).

The results in [10] rigorously establish that the solutions of the NLCME remain asymptotically close to solutions of the original Maxwell equations. This proximity result would appear to be in contradiction with the results just presented in Figures 1 and 2, which show NLCMEd solutions drifting away from the corresponding NLCME solutions because of the destabilization and development of the dispersive scales. There is no contradiction because the result in [10] does not give information about the stability of the solutions involved: when a stable NLCME solution is dispersively unstable, then the result in [10] simply ensures that there is a corresponding close solution of the Maxwell equations, but what happens in the Maxwell equation dynamics is that this solution is also unstable, and the physical system likes to move away from this solution close to the NLCME and develops dispersive scales, as the NLCMEd correctly predict. In other words, this dispersive destabilization is present in the Maxwell equations and is captured by the NLCMEd, but it is simply not seen in the framework of the zero-dispersion NLCME. This point was also numerically verified in [17], where the solutions of the NLCMEd and the Maxwell equations were computed and compared.

In the derivation of the NLCMEd (1.3)-(1.4) from the Maxwell-Lorentz equations, no relative scaling among the different small parameters has to be assumed. To make things clear, we briefly sketch below this derivation procedure without scaling. (A detailed derivation 

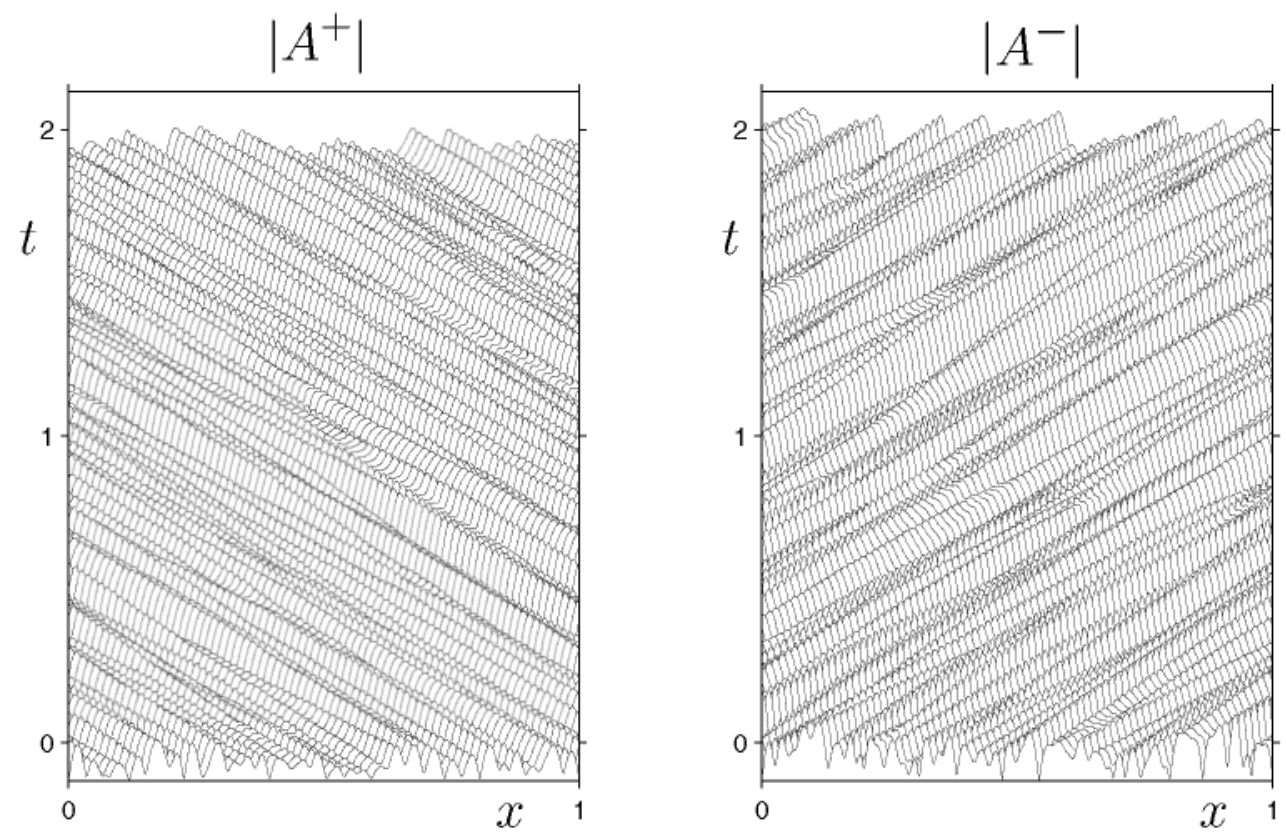

Figure 2. Space-time representation of a solution of the NLCMEd exhibiting small dispersive scales all over the domain $\left(\sigma=1 / 2, \kappa=2, \varepsilon=-10^{-3} / 4\right.$, and periodicity boundary conditions). See the accompanying file (69822_02.gif [20.1MB]) for an animation of the onset of the dispersive scales.

can be found in [17], and a similar derivation in the context of the oscillatory instability in extended dissipative systems is given in [19].)

The Maxwell-Lorentz equations for the original physical problem of light propagation in an FBG can be written as (using the same nondimensionalization as in [10])

$$
\begin{aligned}
& \frac{\partial^{2}(E+P)}{\partial \tilde{t}^{2}}=\frac{\partial^{2} E}{\partial \tilde{x}^{2}}, \\
& \frac{\partial^{2} P}{\partial \tilde{t}^{2}}=-\omega_{p}^{2}(1-2 \Delta n \cos (2 \tilde{x})) P+\omega_{p}^{2}\left(n_{0}^{2}-1\right) E+\omega_{p}^{2} P^{3},
\end{aligned}
$$

where the only small parameter is the intensity of the grating $\Delta n \sim \varepsilon \ll 1$. The basic spatial scale of the periodic grating is $\lambda_{g}=\pi$, and the rest of the parameters are of order 1 and independent of $\varepsilon$. We look for weakly nonlinear solutions that are a superposition of the grating resonant wavetrains

$$
(E, P) \sim\left(\tilde{A}^{+} e^{i \tilde{x}+i \omega \tilde{t}}+\tilde{A}^{-} e^{-i \tilde{x}+i \omega \tilde{t}}\right)+\text { c.c. }+\cdots,
$$

where c.c. stands for complex conjugate. The only assumptions required to obtain the envelope equations are

$$
\cdots \ll\left|\tilde{A}_{\tilde{x} \tilde{x}}^{ \pm}\right| \ll\left|\tilde{A}_{\tilde{x}}^{ \pm}\right| \ll\left|\tilde{A}^{ \pm}\right| \ll 1, \quad \cdots \ll\left|\tilde{A}_{\tilde{t}}^{ \pm}\right| \ll\left|\tilde{A}^{ \pm}\right| \ll 1, \quad \text { and } \quad \Delta n \sim \varepsilon \ll 1,
$$

that is, small amplitudes that depend slowly on space and time, and small grating strength. The expressions above are inserted into (1.5)-(1.6), and an expansion in powers of the small 
quantities $\Delta n, \tilde{A}^{ \pm}, \tilde{A}_{\tilde{x}}^{ \pm}, \tilde{A}_{\tilde{x} \tilde{x}}^{ \pm}, \ldots$ is obtained. After applying solvability conditions to the resonant problems at each order, we obtain the terms of the resulting envelope equations. We now retain the first order nonlinear terms, the first order effect of the grating, and the first two linear effects (transport and dispersion), and, after rescaling $\tilde{x}, \tilde{t}$, and $\tilde{A}^{ \pm}$as in [10], we end up with the NLCMEd equations (1.3)-(1.4).

Note that if one sets a priori the scaling $\tilde{x}, \tilde{t} \sim 1 / \varepsilon$ and $\left|\tilde{A}^{ \pm}\right| \sim \sqrt{\varepsilon}$, then the standard NLCME (1.1)-(1.2) are obtained. However, if we try a dispersive nonlinear Schrödinger (NLS)-like scaling, $\tilde{x} \sim 1 / \sqrt{\varepsilon}$ and $\left|\tilde{A}^{ \pm}\right| \sim \sqrt{\varepsilon}$, then at first order just propagation with the group velocity for times $\tilde{t} \sim 1 / \sqrt{\varepsilon}$ is obtained. Also, if the length of the domain is of order $L \sim 1 / \sqrt{\varepsilon}$ and we have periodicity boundary conditions, one ends up, in the longer time scale $\tilde{t} \sim 1 / \varepsilon$, with two averaged NLS equations like the ones analyzed in [15] and [18]. On the other hand, the NLCMEd analyzed in this paper allow us to cover a more general problem: the onset, in the standard NLCME scenario with typical spatial scale $\tilde{x} \sim 1 / \varepsilon$, of small dispersive scales $\tilde{x} \sim 1 / \sqrt{\varepsilon}$. In this case, all scales in between those two can appear, and in fact they do appear, as is shown in Figures 1 and 2.

The main goal of this paper is to show that, in addition to complex spatio-temporal patterns, the dispersion effects can also give rise to new, purely dispersive localized states, which might be of interest from the optical communications point of view. It is interesting to note that the results in this paper apply also to Bose-Einstein condensates in optical lattices (a system that has recently received very much attention $[22,25])$ and, in general, to any dissipationless propagative system, extended in one spatial direction, reflection- and translation invariant, and with a small superimposed spatial periodic modulation of its background, since the NLCMEd are the appropriate envelope equations for the description of the weakly nonlinear resonant dynamics of this kind of system.

In order to show that light propagation in an FBG can happen in the form of dispersive pulses, in section 2 we derive and solve numerically an asymptotic equation for a family of symmetric pulses, and in section 3 we perform some numerical integrations of the complete NLCMEd to show that some of the pulses in this family do propagate as stable localized structures. Finally, some concluding remarks are drawn in section 4.

2. Dispersive pulses. The starting point is the continuous wave (CW) family of constant uniform modulus solutions of the NLCME (1.1)-(1.2),

$$
\begin{aligned}
& A_{\mathrm{CW}}^{+}=\rho \cos \theta e^{i \omega t+i m x}, \\
& A_{\mathrm{CW}}^{-}=\rho \sin \theta e^{i \omega t+i m x},
\end{aligned}
$$

where $\rho \geq 0$ is the light intensity flowing through the fiber, $\theta \in\left[-\frac{\pi}{2}, \frac{\pi}{2}\right]$ measures the relative amount of both wavetrains, and the frequency and wavenumber of the amplitudes are given by

$$
\begin{gathered}
\omega=\frac{\kappa}{\sin 2 \theta}+\frac{\sigma+1}{2} \rho^{2}, \\
m=\left(\frac{\kappa}{\sin 2 \theta}-\frac{\sigma-1}{2} \rho^{2}\right) \cos 2 \theta .
\end{gathered}
$$




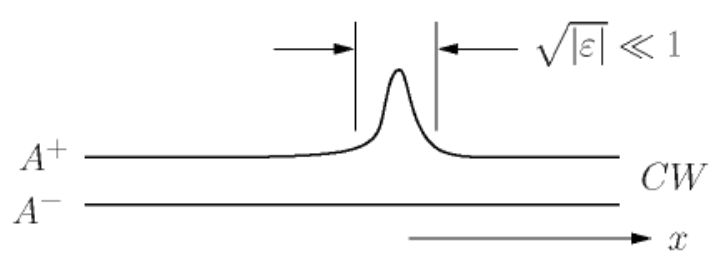

Figure 3. Sketch of a dispersive pulse on top of a continuous wave.

The CWs with $|\omega| \sim 1$ and $|m| \sim 1$ are approximate solutions of the NLCMEd (up to order $\varepsilon$ corrections) and their stability was first analyzed in [8] and then completed in [16], where it was found that, for both signs of the dispersion coefficient, there are dispersively unstable $\mathrm{CW}$ which are stable in the dispersionless context of the NLCME.

The NLCMEd (1.3)-(1.4) have to be considered in the small dispersion limit $\varepsilon \rightarrow 0$. When looking for small-dispersion induced solutions it seems somehow natural to try to proceed in a similar way as in the semiclassical limit of the NLS, i.e., looking for solutions with a fast dispersive phase extended all over the domain (see, e.g., $[4,11]$ ). But in this case, due to the necessary dominance of the transport terms, one obtains at first order just linear propagation with constant group velocity $( \pm 1)$ for the phases, and therefore the phenomenology of the semiclassical limit of the NLS equation (shocks, singularities, etc.) that comes from the resulting nonlinear equation for the phase is not present in the regime explored here.

Instead, we look here for a different type of solution: small-dispersion induced solutions in which the dispersion effects are not extended but localized. More precisely, we look for dispersive pulses of width $\sim \sqrt{|\varepsilon|}$ that propagate on top of a stable uniform CW, as sketched in Figure 3. In order to turn the background $\mathrm{CW}$ into a constant, it is convenient to first perform in the NLCMEd the change of variables

$$
\begin{aligned}
& A^{+}=F^{+} e^{i \omega t+i m x}, \\
& A^{-}=F^{-} e^{i \omega t+i m x},
\end{aligned}
$$

to obtain

$$
\begin{aligned}
& F_{t}^{+}-F_{x}^{+}+\mathrm{i}(\omega-m) F^{+}=\mathrm{i} \kappa F^{-}+\mathrm{i} F^{+}\left(\sigma\left|F^{+}\right|^{2}+\left|F^{-}\right|^{2}\right)+\mathrm{i} \varepsilon F_{x x}^{+}, \\
& F_{t}^{-}+F_{x}^{-}+\mathrm{i}(\omega+m) F^{-}=\mathrm{i} \kappa F^{+}+\mathrm{i} F^{-}\left(\sigma\left|F^{-}\right|^{2}+\left|F^{+}\right|^{2}\right)+\mathrm{i} \varepsilon F_{x x}^{-} .
\end{aligned}
$$

A localized dispersive pulse on $F^{+}$depends on the fast spatial scale $X=x / \sqrt{|\varepsilon|}$, and, according to $(2.5)-(2.6)$, in the short time scale $T=t / \sqrt{|\varepsilon|} \sim 1$ it just propagates with the group velocity, suggesting that we have to look for solutions of the form

$$
\begin{aligned}
& F^{+}=F_{0}^{+}(\eta, t)+\cdots, \\
& F^{-}=F_{0}^{-}(\eta, t)+\cdots,
\end{aligned}
$$

with $\eta=X+T$. Inserting the above ansatz into (2.5)-(2.6) yields

$$
F_{0 \eta}^{-}=0, \quad \text { which gives } F_{0}^{-}=\rho \sin \theta \text {, }
$$


which means that $F^{-}$remains in first approximation equal to the unperturbed CW. Similarly, the following equation is obtained for $F_{0}^{+}$:

$$
F_{0 t}^{+}+\mathrm{i}(\omega-m) F_{0}^{+}=\mathrm{i} \kappa \rho \sin \theta+\mathrm{i} F_{0}^{+}\left(\sigma\left|F_{0}^{+}\right|^{2}+\rho^{2} \sin ^{2} \theta\right) \pm \mathrm{i} F_{0 \eta \eta}^{+},
$$

where the $+(-)$ sign corresponds to $\varepsilon$ positive (negative), together with the boundary conditions

$$
F_{0}^{+} \rightarrow \rho \cos \theta \text { for } \eta \rightarrow \pm \infty,
$$

which ensure that the background $\mathrm{CW}$ is recovered away from the pulse.

For dispersive pulses propagating over a zero background we have to set to zero $\rho, \omega$, and $m$ in (2.7). A standard NLS equation is then obtained, which is known to exhibit localized pulses (solitons) in the focusing case of positive dispersion (recall that $\sigma>0$ ). Note that the effect of the grating on the dispersive pulses is felt only through the background $\mathrm{CW}$, and therefore it is completely gone in this case. In the scaling of the original problem of light propagation on an FBG ((1.5)-(1.6)), the only nonzero Fourier spectrum components of these NLS solitons correspond, in first approximation, to dispersive wavenumbers $\left(\tilde{k}=\tilde{k}_{\text {resonant }}+\Delta \tilde{k}\right.$, with $\Delta \tilde{k} \sim \sqrt{|\varepsilon|}$ ) that are so off-resonance that they simply do not feel the grating (recall that $\tilde{k}_{\text {resonant }}= \pm 1$; see $\left.(1.7)\right)$.

Equation (2.7) is an NLS equation with a direct forcing term coming from the effect of the grating. The steady solutions of this equation and their stability properties were analyzed in $[2,3]$, where explicit analytic expressions for the steady pulses were found. In this paper we consider the more general family of traveling localized solutions. More precisely, we look for traveling pulses of the form

$$
F_{0}^{+}=\rho \cos \theta(1+a(\eta+v t))
$$

where $v$ represents, in the original variables, a small correction of the group velocity. The resulting boundary value problem for $a$, after making use of relations (2.3) and (2.4) and the rescaled variable $\xi=(\eta+v t)(\sqrt{\sigma} \rho \cos \theta)$, can be written as

$$
\begin{gathered}
a_{\xi \xi}=-i \hat{v} a_{\xi}+\alpha a-\left(|a|^{2}+a+\bar{a}\right)(1+a), \\
a \rightarrow 0 \text { as } \xi \rightarrow \pm \infty,
\end{gathered}
$$

where $\hat{v}=v /(\sqrt{\sigma} \rho \cos \theta)$ and $\alpha=k \tan (\theta) /\left(\sigma \rho^{2} \cos ^{2} \theta\right)$. We are restricting our search to the focusing case of positive sign in (2.7) and, in order to have a dispersively stable background $\mathrm{CW}$, we have to consider only the range $0<\theta<\frac{\pi}{2}$ (see [16]), which implies that we have to look for pulses in (2.9)-(2.10) only for $\alpha>0$. On the other hand, (2.9)-(2.10) remain invariant under the transformation $\hat{v} \rightarrow-\hat{v}$ and $a \rightarrow \bar{a}$, and therefore we can also set $\hat{v} \geq 0$.

The solutions of (2.9)-(2.10) correspond to traveling dispersive pulses and can be regarded as homoclinic orbits (in the variable $\xi$ ) connecting the trivial state (i.e., $a=0$ ) back to itself.

To analyze the existence of such connections we first consider the linearized system around the zero state:

$$
\frac{d \mathbf{u}}{d \xi}=A_{\infty} \mathbf{u},
$$




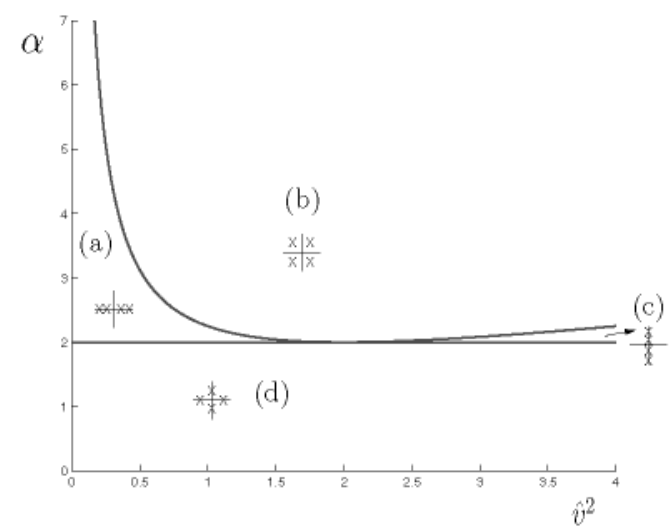

Figure 4. Eigenvalues of the trivial state as a function of the parameters $\alpha$ and $\hat{v}^{2}$. Sketches show the distribution of the four eigenvalues in each of the distinct regions.

where $\mathbf{u}^{T} \equiv\left(a_{r}, a_{i},\left(a_{r}\right)_{\xi},\left(a_{i}\right)_{\xi}\right), a_{r}$ and $a_{i}$ are the real and imaginary part of $a,\left(a_{r}\right)_{\xi}=d a_{r} / d \xi$ and $\left(a_{i}\right)_{\xi}=d a_{i} / d \xi$, and the matrix $A_{\infty}$ is given by

$$
A_{\infty}=\left(\begin{array}{cccc}
0 & 0 & 1 & 0 \\
0 & 0 & 0 & 1 \\
\alpha-2 & 0 & 0 & \hat{v} \\
0 & \alpha & -\hat{v} & 0
\end{array}\right) .
$$

The eigenvalues of this system are given by $\lambda_{ \pm}^{1}=\sqrt{\eta_{ \pm}}$and $\lambda_{ \pm}^{2}=-\sqrt{\eta_{ \pm}}$, with

$$
\eta_{ \pm}=\left[2 \alpha-\left(\hat{v}^{2}+2\right) \pm \sqrt{\left(\hat{v}^{2}+2\right)^{2}-4 \hat{v}^{2} \alpha}\right] / 2 .
$$

Figure 4 shows the behavior of the four eigenvalues $\lambda_{ \pm}^{1}$ and $\lambda_{ \pm}^{2}$ in the $\left(\alpha, \hat{v}^{2}\right)$ plane. There are four distinct regions, separated by the boundaries $\alpha=2$ and $\alpha=\left(\hat{v}^{2}+2\right)^{2} / 4 \hat{v}^{2}$ : (a) four real eigenvalues, (b) two pairs of complex conjugate eigenvalues, (c) four purely imaginary eigenvalues, and (d) two real eigenvalues along with two purely imaginary eigenvalues. In regions (a) and (b) the unstable and stable manifolds of the origin are two dimensional, while the equilibrium is nonhyperbolic in regions (c) and (d) where the center manifold is, respectively, four- and two dimensional. Homoclinic orbits belong to both the stable and unstable manifold of the origin. We investigate below the presence of homoclinic solutions in cases (a) and (b), where these correspond to the intersections of two dimensional stable and unstable manifolds in a four dimensional space [23].

The problem (2.9)-(2.10) is invariant under the symmetry

$$
a \rightarrow \bar{a}, \quad \xi \rightarrow-\xi,
$$

that comes from the time reversing (Hamiltonian) and spatial reflection symmetries of the NLCMEd. We further restrict our search for dispersive pulses to the case of reflectionsymmetric pulses, i.e., to pulses that satisfy

$$
a(\xi)=\bar{a}(-\xi) .
$$


If we now set the symmetry axis to $\xi=0$, we can reduce the problem to a semi-infinite interval $\xi \in[0,+\infty[$ with the boundary conditions

$$
\begin{aligned}
& \left(a_{r}, a_{i}\right)=\left(a_{0}, 0\right) \text { and }\left(\left(a_{r}\right)_{\xi},\left(a_{i}\right)_{\xi}\right)=\left(0, b_{0}\right) \text { at } \xi=0, \\
& \left(a_{r}, a_{i}\right) \rightarrow(0,0) \text { as } \xi \rightarrow \infty .
\end{aligned}
$$

Finally, to numerically compute the profiles of the symmetric pulses, we replace the infinite interval by a finite one, $[0, L]$. Following [13], the resulting boundary conditions at $\xi=L$ are obtained by requiring that the solution be in the subspace spanned by the eigenvectors associated with the decaying eigenvalues of the matrix $A_{\infty}$ (see (2.11)). In summary, the boundary value problem that we integrate numerically is given by (2.9), which we rewrite as a real first order system of four equations in $] 0, L[$ together with the four boundary conditions

$$
\begin{aligned}
C_{0} \mathbf{u} & =0 \quad \text { at } \quad \xi=0 \quad \text { and } \\
C_{\infty} \mathbf{u} & =0 \quad \text { at } \quad \xi=L,
\end{aligned}
$$

where $\mathbf{u}^{T} \equiv\left(a_{r}, a_{i},\left(a_{r}\right)_{\xi},\left(a_{i}\right)_{\xi}\right)$,

$$
C_{0}=\left(\begin{array}{cccc}
0 & 1 & 0 & 0 \\
0 & 0 & 1 & 0
\end{array}\right)
$$

and $C_{\infty}$ is a matrix whose rows are the left eigenvectors of $A_{\infty}$ associated with the exponentially growing directions

$$
C_{\infty}=\left(\begin{array}{llll}
\left(\eta_{+}-\alpha-\hat{v}^{2}\right) & \alpha \hat{v} / \sqrt{\eta_{+}} & \left(\eta_{+}-\alpha\right) / \sqrt{\eta_{+}} & \hat{v} \\
\left(\eta_{-}-\alpha-\hat{v}^{2}\right) & \alpha \hat{v} / \sqrt{\eta_{-}} & \left(\eta_{-}-\alpha\right) / \sqrt{\eta_{-}} & \hat{v}
\end{array}\right) .
$$

For each value of $\alpha$ this problem is solved using a shooting method. We start from the known solutions for $\hat{v}=0$ obtained in [3] and apply numerical continuation techniques to locate the propagating pulses with $\hat{v}>0$. This procedure for setting the boundary conditions at $\xi=L$, rather than simply imposing $a(L)=0$, allows the shooting method to converge faster, and the results obtained are found to be essentially independent of $L$ for $L \gtrsim 10$.

The left panel of Figure 5 shows several families of homoclinic orbits represented in the $\left(a_{0}, \hat{v}\right)$ plane for different values of $\alpha$ and corresponding to the case $a_{0}>0$. The dashed line separates the regions where the homoclinic orbit connects to a saddle point and where it connects to a saddle-focus, while the open circles correspond to the solutions shown in the right panels. In Figure 5(II)(a)-(c) the solutions can be seen to develop oscillations as we move towards $a_{0}=0$. This corresponds to moving along a horizontal line in Figure 4 (constant $\alpha$ ) and to the right, approaching region (c) (precisely at $a_{0}=0, \hat{v}=\sqrt{\alpha}+\sqrt{\alpha-2}$ ) where the eigenvalues become purely imaginary.

This oscillatory behavior, however, is not observed for the families of pulse solutions found for $a_{0}<0$, as seen in Figure 6 . Instead, these curves display turning points and, to better appreciate these limit points, the results have been plotted in the plane $(E, \hat{v})$, where $E$ is the positive quantity

$$
E=\sqrt{\int_{0}^{\infty}\left(|a|^{2}+\left|a_{\xi}\right|^{2}\right) d \xi}
$$

In this case, as we move along the curves for fixed $\alpha$ and past the turning point, the pulses develop two extra humps that tend to move away from the origin (see Figure 6 (II) (a) and (b)). 
(a)

(I)

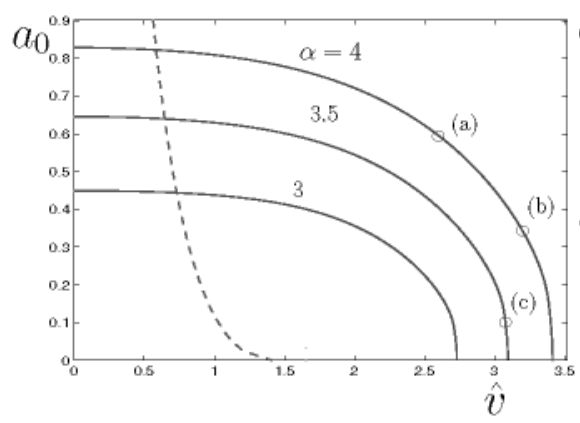

(b)

(II)
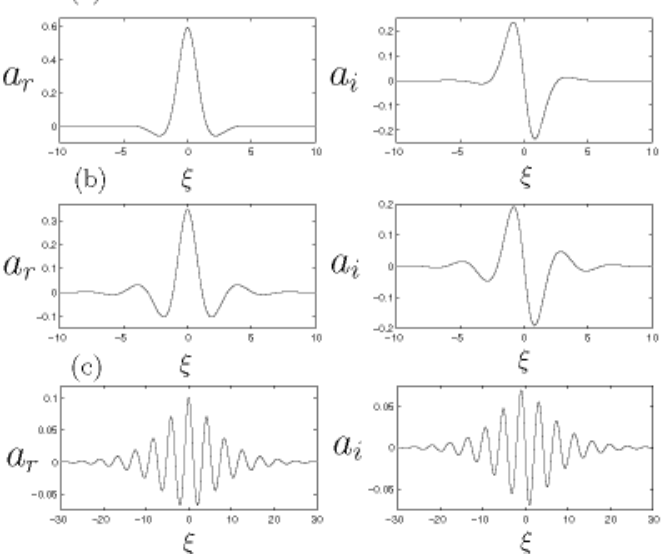

Figure 5. (I) The solid lines correspond to homoclinic cycles to the origin with $a_{0}>0$ for the indicated values of $\alpha$. The dashed line bounds the regions where the origin is a saddle node (on the left) and saddle-focus (on the right). (II) Spatial profiles of three pulses for the values marked in (I) with open circles.

(a)

(I)

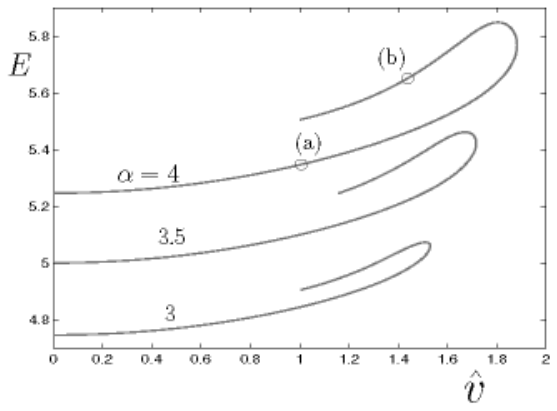

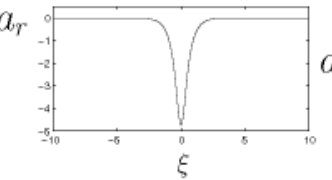

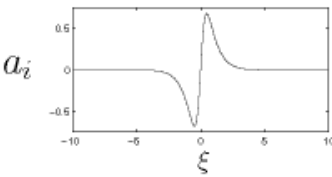

(b)
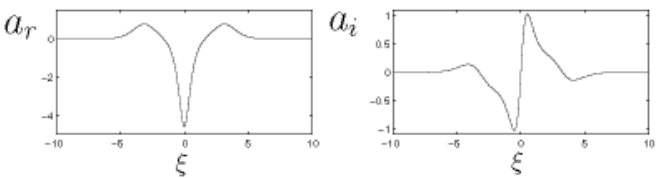

Figure 6. (I) Curves of homoclinic cycles to the origin with $a_{0}<0$ for the indicated values of $\alpha$. (II) Spatial profiles of two pulses corresponding to the values marked in (I) with open circles.

3. NLCMEd simulations. After having found a two-parameter $(\alpha, \hat{v})$ family of symmetric dispersive pulses (DP) that can be numerically continued from the solutions for $\hat{v}=0$ obtained in [3], we now proceed to study their stability.

The idea is not to perform a complete stability analysis of the family of DP, but to show that stable DP can be found and that the DP can thus be considered as robust realizable localized structures of light propagation in FBG. To do this we select several DP, place them on top of their corresponding background $\mathrm{CW}$, add a small random perturbation, and use them as initial conditions for the full system of NLCMEd (1.3)-(1.4) that we numerically integrate for a certain amount of time with periodic boundary conditions. The numerical method for the integration of the NLCMEd uses a Fourier series in space with $N_{F}$ modes and a fourth order Runge-Kutta scheme for the time integration of the resulting system of ordinary differential equations for the Fourier coefficients. The stiff linear diagonal terms 

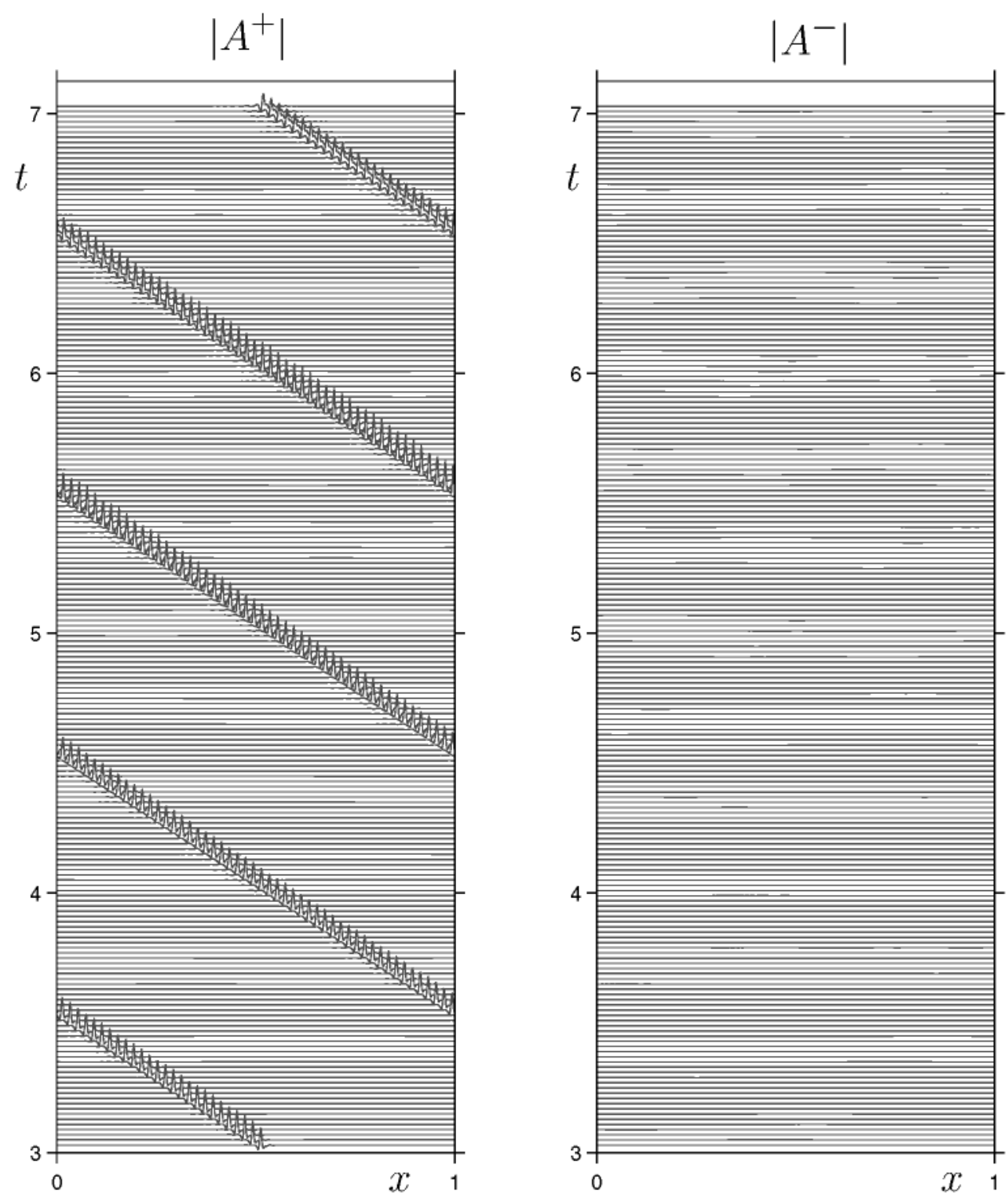

Figure 7. Space-time representation of the solution of the NLCMEd $\left(\sigma=1 / 2, \kappa=1, \varepsilon=10^{-5}\right.$, and periodicity boundary conditions) for an unstable pulse propagating on top of a $C W(\rho=1$ and $\theta=\pi / 4)$. The pulse parameters are $\alpha=4$ and $\hat{v}=1$, and it corresponds to the point labeled (a) in Figure 6. See the accompanying file (69822_03.gif [15.8MB]) for an animation of this pulse destabilization.

associated with the small dispersion coefficients are integrated implicitly, and the nonlinear terms are computed in physical space with the usual $2 / 3$ rule to avoid aliasing effects [5]. (The maximum required resolution for the simulations in this paper was $N_{F}=4096$ and $\Delta t=.0005$.)

Unstable DP simply do not persist, and their shape changes, as can be appreciated from the spatio-temporal evolution shown in Figure 7, which corresponds to the pulse labeled (a) in 

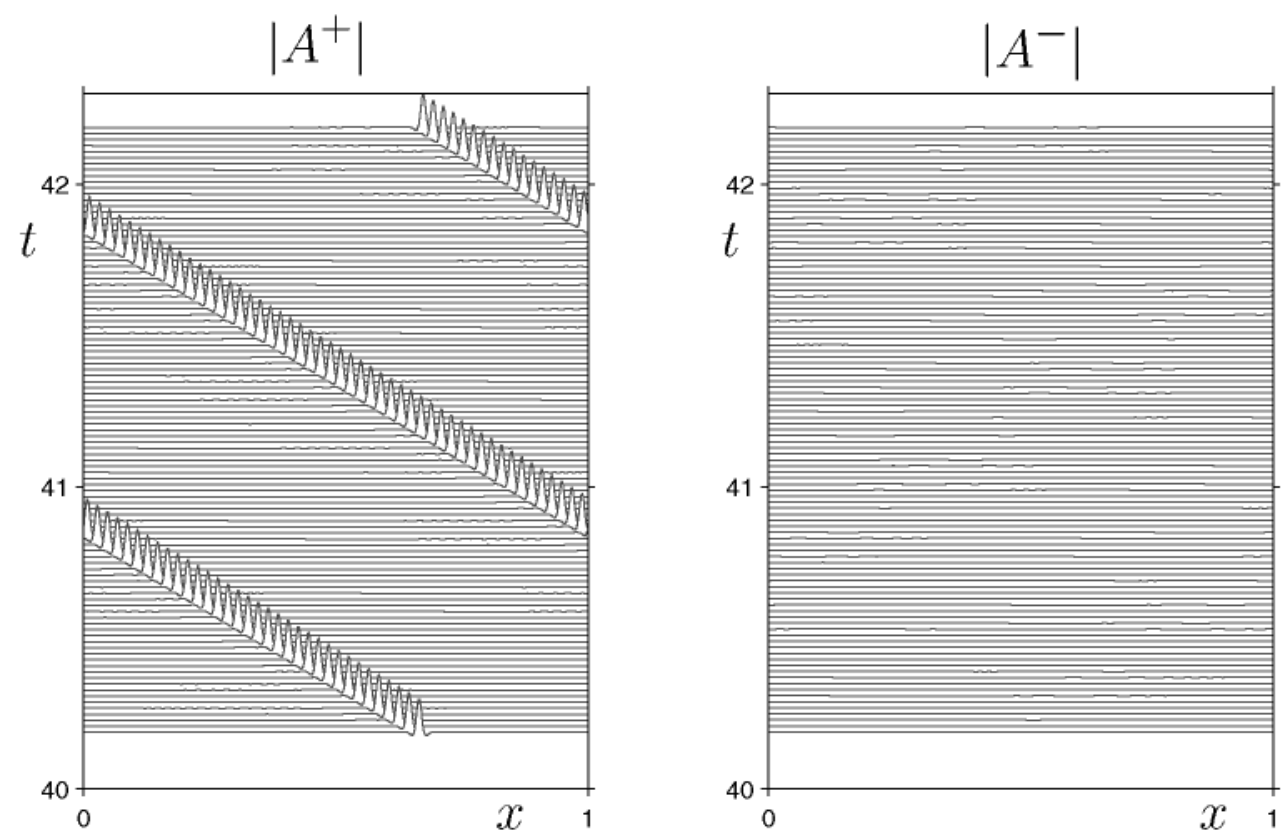

Figure 8. Space-time representation of the solution of the $\operatorname{NLCMEd}\left(\sigma=1 / 2, \kappa=1, \varepsilon=10^{-5}\right.$, and periodicity boundary conditions) for a stable pulse propagating on top of a $C W(\rho=1$ and $\theta=\pi / 4)$. The pulse parameters are $\alpha=4$ and $\hat{v}=2.6$, and it corresponds to the point labeled (a) in Figure 5 . See the accompanying file (69822_04.gif [3.6MB]) for an animation of this propagating pulse.

Figure 6 (see also the corresponding animation 69822_03.gif [15.8MB]). Note that the size of the pulse first grows (at $t=5.5$ it is larger than at $t=3$ ), and then it decays again at $t=7.0$. A few time units later the oscillatory tails spread, and the pulse structure is eventually lost (not shown in the figure). All DP explored for $a_{0}<0$ propagated over the same simple CW with parameters $\rho=1$ and $\theta=\pi / 4$ (cf. (2.1) $-(2.2)$ ), which corresponds to $\alpha=4$ in (2.14), and all were found to be unstable regardless of the propagation speed $\hat{v}$.

On the other hand, for $a_{0}>0$ and for the same background CW (i.e., $\alpha=4$ ), the DP are found to be unstable approximately for $0 \leq \hat{v} \lesssim 2.2$ and stable for $v \gtrsim 2.2$. The evolution of two stable pulses is shown in Figures 8 and 9, which correspond to $\hat{v}=2.6$ and $\hat{v}=3.2$, respectively, where the structure of the slightly perturbed pulses is seen to remain now virtually unaltered after more than 40 time units (see also the corresponding animations 69822_04.gif [3.6MB] and 69822_05.gif [3.5MB]).

The stability of the pulses was tested by numerical simulation of the complete envelope equations (1.3)-(1.4), using as initial condition the pulse with a small superimposed random perturbation (the details of the spectral numerical integration scheme are given in [16]). For the case of stable pulses they remained practically undistorted for times of the order of $t=40$ (Figures 8 and 9). This is a sufficiently large time since the growth rate of the instabilities is clearly of order 1, as can be seen in Figure 7 where the unstable pulses are already severely distorted at $t=7$. Despite the fact that this is not a rigorous stability analysis, we believe that the results of these simulations strongly suggest that some of the pulses found are indeed stable and are therefore robust dynamical states of the system for the time scale $t \sim 1$, which 

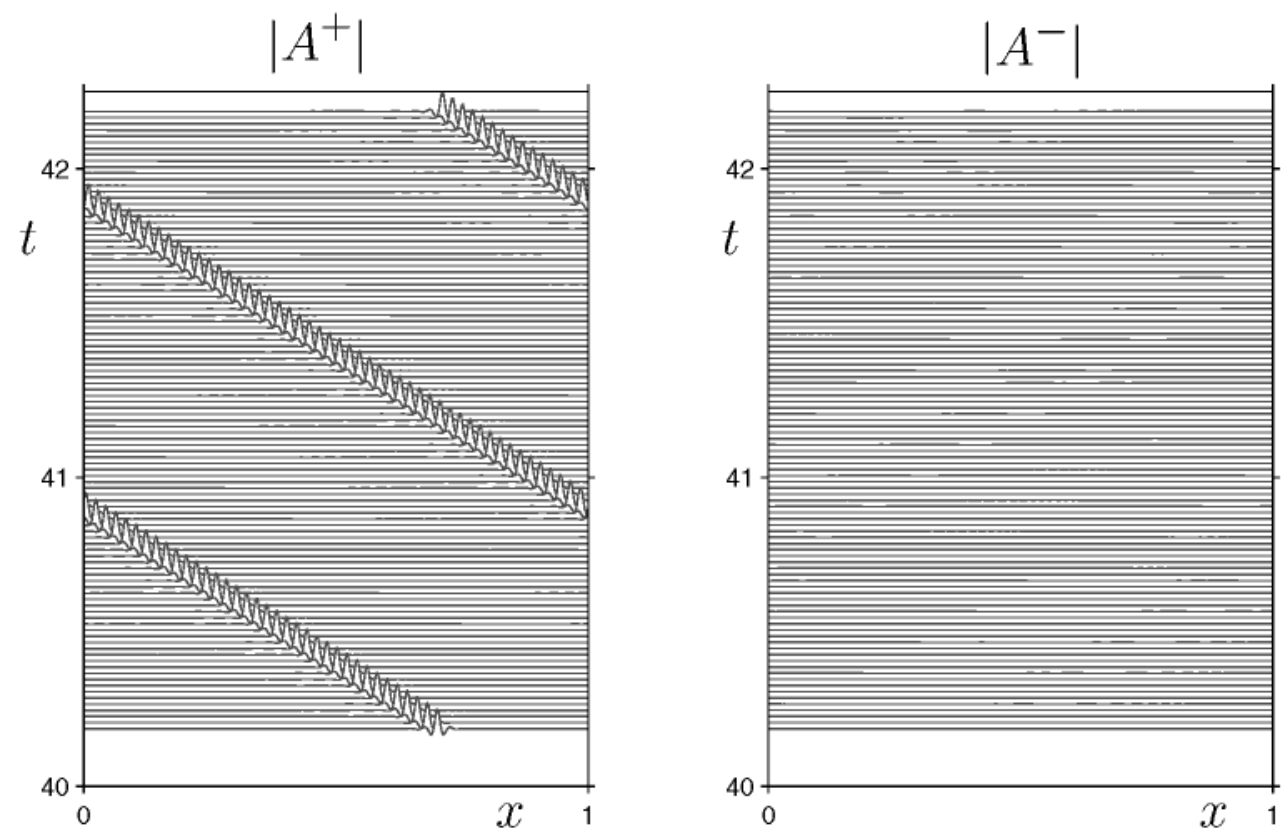

Figure 9. Space-time representation of the solution of the $\operatorname{NLCMEd}\left(\sigma=1 / 2, \kappa=1, \varepsilon=10^{-5}\right.$, and periodicity boundary conditions) for a stable pulse propagating on top of a $C W$ ( $\rho=1$ and $\theta=\pi / 4)$. The pulse parameters are $\alpha=4$ and $\hat{v}=3.2$, and it corresponds to the point labeled (b) in Figure 5 . See the accompanying file (69822_05.gif [3.5MB]) for an animation of this propagating pulse.

is the relevant one in the NLCME dynamics.

Another very interesting feature of the DP that is worth mentioning is the fact that they are somehow transparent to each other: two DP propagating in opposite directions just pass through each other without distortion. This is illustrated in Figure 10 (see also the animation 69822_06.gif [3.9MB]), where two stable DP (corresponding to those in Figures 8 and 9) are sent towards each other and after 40 time units (approximately 80 collisions) they still remain practically undistorted. The reason behind this behavior is the dominant character of the transport effect induced by the group velocity. If we rewrite the NLCMEd for a DP with short (dispersive) spatial and temporal scales $X=x / \sqrt{\varepsilon} \sim 1$ and $T=t / \sqrt{\varepsilon} \sim 1$, they take, at first order, the form of two uncoupled wave equations:

$$
\begin{gathered}
A_{T}^{+}=A_{X}^{+}+\cdots, \\
A_{T}^{-}=-A_{X}^{-}+\cdots .
\end{gathered}
$$

It is clear then that the DP traveling in opposite directions simply propagate through different channels, and are (in first approximation) completely independent.

4. Conclusions. In this paper we have studied the effect of dispersion in the weakly nonlinear dynamics of light propagation in an FBG. We have shown that the (often neglected) small dispersion terms play a crucial role in the transport-dominated dynamics of light propagation in FBG. The combined effect of propagation at the group velocity and dispersion can give rise to complex spatio-temporal chaotic states, but also to a new family of localized 

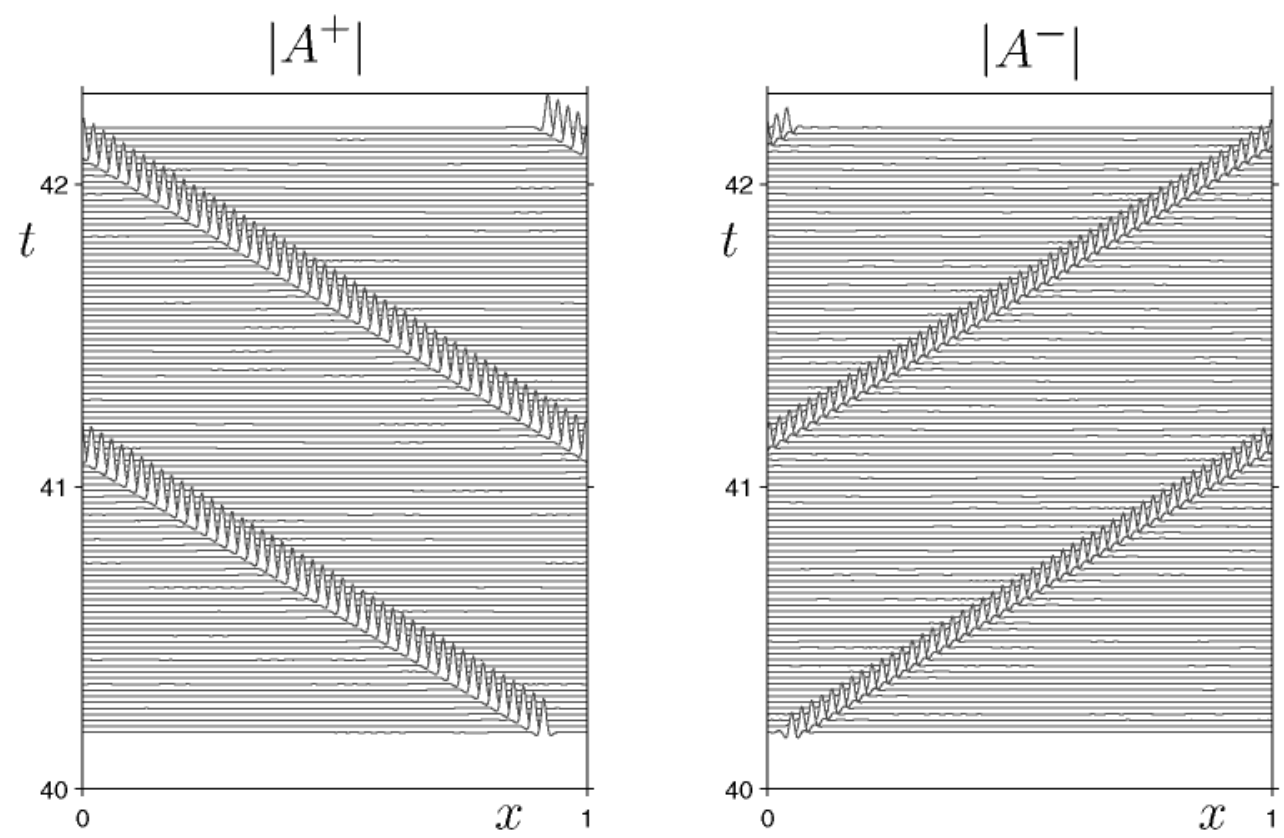

Figure 10. Space-time representation of the solution of the $\operatorname{NLCMEd}\left(\sigma=1 / 2, \kappa=1, \varepsilon=10^{-5}\right.$, and periodicity boundary conditions) showing the simultaneous propagation in opposite directions of the two stable pulses from Figures 8 and 9. See the accompanying file (69822_06.gif [3.9MB]) for an animation of this pulse interaction.

states (dispersive pulses, DP) that propagate on top of a CW. These DP are approximately advected by the group velocity, but this transport effect does not play any role in the determination of their internal structure, which results basically from a balance between nonlinearity and dispersion. It is also important to emphasize that this type of dynamics is not contained in the standard dispersionless NLCME (1.1)-(1.2) formulation usually applied to model light propagation in FBG.

In the context of light propagation on FBG the localized structures that have been most extensively analyzed are the so-called gap solitons (GS). The GS are well-known pulse-like solutions of the NLCME (1.1)-(1.2) that have the striking property that they can propagate at any velocity between zero and the speed of light of the bare fiber (see, e.g., $[1,10]$ and references therein). The new family of localized structures presented in this paper, the DP, appear in the same regime of weakly nonlinear light propagation in FBG where the GS exist (the most frequently analyzed regime). There are robust stable DP, and their dynamics evolve in the same time scale as the GS, but they exhibit several essentially different characteristics that are worth mentioning: (i) the GS propagate over the zero intensity state, while the DP propagate on top of a saturated uniform continuous wave; (ii) both GS and DP have amplitudes of the same size, but the DP are much more narrow pulses (in the scalings used in this paper, $\delta_{\mathrm{DP}} \sim \delta_{\text {disp }} \sim \sqrt{\varepsilon} \ll 1$ and $\delta_{\mathrm{GS}} \sim 1$ ); (iii) the DP propagate with the speed of light of the bare fiber $(v= \pm 1)$ and only on one of the amplitudes, $A^{+}$or $A^{-}$, while the GS have nonzero component on both amplitudes; and, finally, (iv) the DP have the property that two DP propagating in opposite directions just pass through each other without distortion, which, 
a priori, looks like it could be interesting from the point of view of information transmission.

The ideas used in the analysis performed in this paper go beyond the context of light propagation on fiber gratings and apply to the (systematically not considered in the literature) generic case of pattern formation in propagative spatially extended systems. The main ingredients are (i) the unavoidable different asymptotic order of transport (first order spatial derivatives) and dispersion/dissipation (second derivatives), and (ii) the need to consider these two effects simultaneously in order to correctly account for the weakly nonlinear dynamics of the system. The envelope equations are necessarily asymptotically nonuniform; that is, the small parameter is not gone from the envelope equation formulation, reflecting the impossibility of balancing the two effects mentioned above. The resulting dynamics is much richer, and new patterns (as the pulses obtained in this paper) and instabilities can be found that have shorter spatial scales but that are still slow as compared with the basic linear wavetrains and therefore well accounted for by the envelope equation formalism. All these new dynamical states are not detected if the usual formulation that only takes into account transport effects is applied. Finally, it is also interesting to mention that similar effects have been previously described in the context of the oscillatory instability in dissipative systems [20] and in parametrically forced surface waves [21].

\section{REFERENCES}

[1] A. Aceves, Optical gap solitons: Past, present and future; theory and experiment, Chaos, 10 (2000), pp. $584-589$.

[2] I. BarashenKov AND Y. S. SMIRNOV, Existence and stability chart for the AC-driven, damped nonlinear Schroedinger solitons, Phys. Rev. E, 54 (1996), pp. 5707-5725.

[3] I. Barashenkov, T. Zhanlav, AND M. Bogdan, Instabilities and soliton structures in the driven nonlinear Schroedinger equation, in Nonlinear World Vol. 1, Proceedings of the IV International Workshop on Nonlinear and Turbulent Processes in Physics, V. Bar'yakhtar, V. Chernousenko, N. Erokhin, A. Sitenko, and V. Zakharov, eds., World Scientific, River Edge, NJ, 1989, pp. 3-9.

[4] J. C. Bronsky and D. W. McLaughuin, Semiclassical behavior in the NLS equation: Optical shocksfocusing instabilities, in Singular Limits of Dispersive Waves, NATO Adv. Sci. Inst. Ser. B Phys. 320, N. Ercolani, I. Gabitov, C. Levermore, and D. Serre, eds., Plenum, New York, 1994, pp. 21-38.

[5] C. Canuto, H. Hussani, A. Quarteroni, And T. Zang, Spectral Methods in Fluid Mechanics, Springer Ser. Comput. Phys, Springer-Verlag, New York, Berlin, 1988.

[6] A. Champneys, B. Malomed, and M. Friedman, Thirring solitons in the presence of dispersion, Phys. Rev. Lett., 80 (1998), pp. 4169-4172.

[7] D. Christodoulides And R. Joseph, Slow Bragg solitons in nonlinear periodic structures, Phys. Rev. Lett., 62 (1989), pp. 1746-1749.

[8] C. DE STERKE, Theory of modulational instability in fiber Bragg gratings, J. Opt. Soc. Amer. B Opt. Phys., 15 (1998), pp. 2660-2667.

[9] C. De Sterke and J. Sipe, Gap solitons, Progr. Optics, 33 (1994), pp. 203-260.

[10] R. Goodman, M. Weinstein, AND P. Holmes, Nonlinear propagation of light in one-dimensional periodic structures, J. Nonlinear Sci., 11 (2001), pp. 123-168.

[11] S. Jin, C. Levermore, AND D. W. McLaUghlin, The behavior of solutions of the NLS equation in the semiclassical limit, in Singular Limits of Dispersive Waves, NATO Adv. Sci. Inst. Ser. B Phys. 320, N. Ercolani, I. Gabitov, C. Levermore, and D. Serre, eds., Plenum, New York, 1994, pp. 235-255.

[12] R. Kashyap, Fiber Bragg Gratings, Optics and Photonics, Academic Press, New York, 1999.

[13] H. Keller, Numerical Solution of Two Point Boundary Value Problems, CBMS-NSF Regional Conf. Ser. in Appl. Math. 24, SIAM, Philadelphia, 1976.

[14] J. Kevorkian and J. Cole, Multiple Scale and Singular Perturbation Methods, Appl. Math. Sci. 114, Springer-Verlag, New York, Berlin, 1996. 
[15] E. KNoBoch AND J. GIBBon, Coupled NLS equations for counterpropagating waves in systems with reflection symmetry, Phys. Lett. A, 154 (1991), pp. 353-356.

[16] C. MARTEL, Dispersive destabilization of nonlinear light propagation in fiber Bragg gratings, Chaos, 15 (2005), paper 013701

[17] C. Martel And C. CASas, Dispersive destabilization of nonlinear light propagation in fiber Bragg gratings: A numerical verification, Chaos, 17 (2007), paper 013114.

[18] C. MARTEL, E. KNOBOCH, AND J. VEGA, Dynamics of counterpropagating waves in parametrically driven systems, Phys. D, 137 (2000), pp. 94-123.

[19] C. Martel AND J. VEGA, Finite size effects near the onset of the oscillatory instability, Nonlinearity, 9 (1996), pp. 1129-1171.

[20] C. Martel And J. VEga, Dynamics of a hyperbolic system that applies at the onset of the oscillatory instability, Nonlinearity, 11 (1998), pp. 105-142.

[21] C. MARTEL, J. VEGA, AND E. KNOBOCH, Dynamics of counterpropagating waves in parametrically driven systems: Dispersion vs. advection, Phys. D, 174 (2003), pp. 198-217.

[22] H. SAKAGUCH AND B. MALOMED, Dynamics of positive- and negative-mass solitons in optical lattices and inverted traps, J. Phys. B, 37 (2004), pp. 1443-1459.

[23] S. Wiggins, Global Bifurcation and Chaos, Appl. Math. Ser. 73, Springer-Verlag, New York, Berlin, 1988.

[24] H. Winful And G. CoOperman, Self-pulsing and chaos in distributed feedback bistable optical devices, Appl. Phys. Lett., 40 (1982), pp. 298-300.

[25] A. YUlin AND D. SKRYABIN, Out-of-gap Bose-Einstein solitons in optical lattices, Phys. Rev. E, 67 (2003), paper 023611. 